\title{
Partial Obstruction and Intestinal Bleeding Secondary to a Congenital Duodenal Diverticulum in a Dog
}

\author{
Carla Aparecida Batista Lorigados' ${ }^{1}$, Aline Machado Zoppa ${ }^{2}$, Andre Schiller ${ }^{3}$, lara Tiburcio ${ }^{3}$, \\ Fabio Giusti Calderón ${ }^{4} \&$ Marianna Pantano 5
}

\begin{abstract}
Background: Intestinal diverticulum is an abnormality resulting in the formation of a blind-ended saccular pouch that can be acquired either congenital, true (involving all intestinal layers) or false (involving the mucosa and submucosa), with extraluminal and intraluminal type. In humans, the acquired is more frequent, the colon is the most affected segment followed by duodenum; and majority cases of duodenal diverticula remains asymptomatic, but biliary obstruction, recurrent acute pancreatitis, hemorrhagic ulcer, proximal intestinal obstruction and perforation may occur. The aim of this report is to present a case of a congenital disease in dogs, prone to misdiagnosis due to non-specific clinical signs.

Case: An 8-month-old male Boxer was evaluated due to recurrent hyporexia, vomiting, melena and syncope over three months with signs of a possible intestinal obstruction. Physical examination showed no abnormalities except for pale mucous membranes. Complete blood count revealed anemia and leukocytosis. Platelets and biochemical profiles were normal. Abdominal ultrasound examination indicated a dilated duodenum, measuring approximately $3.36 \mathrm{~cm}$ in diameter, with heterogeneous fluid content and hyperechoic structures with acoustic shadow, peristalsis appeared decreased and non-progressive. The gastrointestinal positive contrast study was performed to better evaluate abnormalities detected at ultrasonography. Images after 30 min of contrast administration demonstrated a marked distension of the duodenum, filled with contrast and a mildly filled stomach displaced to the left. Sixty min after contrast administration a marked distension of the entire duodenum, with tortuous aspect and filled with contrast was seen. The caudal duodenal flexure was connected to a large barium filled saccular structure that measured approximately $7 \mathrm{~cm}$ in diameter, consistent with a duodenal diverticulum. A blood transfusion was performed and surgical treatment indicated. The diverticulum and a small portion of the caudal duodenal segment were resected, an end-side enteroanastomosis was made due to the difference in diameter between intestinal segments. The patient was medicated with sucralfate $(12.5 \mathrm{mg} / \mathrm{kg})$, ranitidine $(2 \mathrm{mg} / \mathrm{kg})$, metronidazole $(25 \mathrm{mg} / \mathrm{kg})$, dipyrone $(25 \mathrm{mg} / \mathrm{kg})$, tramadol $(2 \mathrm{mg} / \mathrm{kg})$ and recovered quickly from surgery. Histopathological examination characterized the diverticular tissue as a true diverticulum by the presence of all intestinal layers. Post-operative and follow-up evaluations showed no recurrence of clinical signs.

Discussion: In veterinary practice, congenital duodenal diverticulum is a rare condition documented in dogs, curiously all Boxers. None of the reported cases in literature had the diagnostic of duodenal diverticulum made exclusively by ultrasonography. Other diagnostic imaging modalities, such as gastrointestinal barium study or endoscopy, were necessary. In one case a diagnostic was made during exploratory laparotomy. The marked dilatation of the duodenal segment impaired ultrasound evaluation, allowing recognition of an obstructive pattern, not the diverticulum itself. At histopathological examination, the diverticular tissue was characterized by a thickened wall with a hypertrophied muscle layer, characterizing a true duodenal diverticulum. The location, breed and age of the dogs affected with duodenal diverticulum were similar in all veterinary cases reported. Dogs presenting signs of gastrointestinal disease and abdominal pain are common in patients referred to ultrasound examination. However, despite the rare reports described, we must consider this affection as a differential diagnosis, whenever Boxer puppies present these clinical signs associated with gastrointestinal bleeding and syncope.
\end{abstract}

Keywords: duodenum, congenital disease, diagnostic imaging, canine.

${ }^{1}$ Department of Surgery, Faculty of Veterinary Medicine and Animal Science (FMVZ), University of São Paulo (USP), São Paulo, SP, Brazil. ${ }^{2}$ Department of Surgery, Faculty of Veterinary Medicine, Anhembi Morumbi University (UAM), São Paulo. ${ }^{3}$ Department of Surgery, Faculty of Veterinary Medicine, Metropolitan Colleges United (FMU), São Paulo. ${ }^{4}$ PetCare Veterinary Hospital \& ${ }^{5}$ EVET Veterinary Specialties, São Paulo. CORRESPONDENCE: C.A.B. Lorigados [clorigados@usp.br] Department of Surgery, FMVZ - USP. Av. Prof. Dr. Orlando Marques de Paiva n. 87. CEP 05508-270 São Paulo, SP, Brazil. 


\section{INTRODUCTION}

Intestinal diverticulum (ID) is an abnormality that results in the formation of a blind-ended saccular pouch in the intestinal wall and can produce signs of gastrointestinal disease. The duodenal type (DD) was primarily described in humans by Chomel in 1710, and there are reports of ID in animals, including dogs, pigs, horses and sheep $[10,13]$.

Duodenal diverticulum can be acquired or congenital, the last contains all intestinal layers, with an extraluminal (EDD) and intraluminal (IDD) type $[10,12,15]$. The origin of IDD seems to be caused by the presence of a luminal membrane due to recanalization failure of the foregut lumen during fetal stages, later peristaltic and feeding pressure extends it, leading to an intraluminal duodenal diverticulum $[1,5,6,11]$. The acquired EDD is more frequent and characterized by herniation of the submucosa and mucosa, through a muscularis defect or a weak spot of the wall, such as an artery entry; it's also named as pseudodiverticulum $[8,10,12]$. The etiopathogenesis of the intestinal congenital diverticulum remains unclear. In humans it may be a remaining pouch of the cecal appendix during embryological stages [12]. The ID is a common finding in gastrointestinal imaging studies, and the most usual location is in the colon, followed by duodenum. Most of the duodenal diverticulum remain asymptomatic, but serious complications as biliary obstruction, recurrent acute pancreatitis, hemorrhagic ulcer, proximal intestinal obstruction and perforation may occur $[2,5,7,14]$.
The aim of this report is to present a rare case of a congenital disease in dogs, prone to misdiagnosis due to non-specific clinical signs.

\section{CASE}

An 8-month-old intact male Boxer was presented to the hospital with recurrent signs of hyporexia, vomiting, melena and syncope over three months. Physical examination showed no abnormalities except for pale mucous membranes. Complete blood count revealed anemia, red blood cells $2.3 \times 10 \% \mu \mathrm{L}$ (reference range 5.0 - 8.0), hematocrit $16 \%$ (reference range 37 $54 \%$ ), and leukocytosis 22,196 uL (reference range 6,000 - 15,000). Platelets and biochemical profiles were normal. Abdominal ultrasound examination indicated a dilated duodenum, measuring approximately $3.36 \mathrm{~cm}$ in diameter, wall thickening with preserved stratification, fluid content and heterogeneous ingesta associated with small hyperechoic structures that produces acoustic shadow (Figure 1). Peristalsis appeared decreased and non progressive within the dilated portion. There were no sonographic changes in other portions of the small intestine and colon.

To better evaluate the abnormalities detected at ultrasonography a gastrointestinal barium study was performed. Radiographs after $30 \mathrm{~min}$ of contrast administration demonstrated a distension of the duodenum, filled with contrast and a mildly filled stomach displaced to the left. Sixty min after contrast administration persisted the marked distension of the duodenum, with tortuous aspect. The caudal duodenal

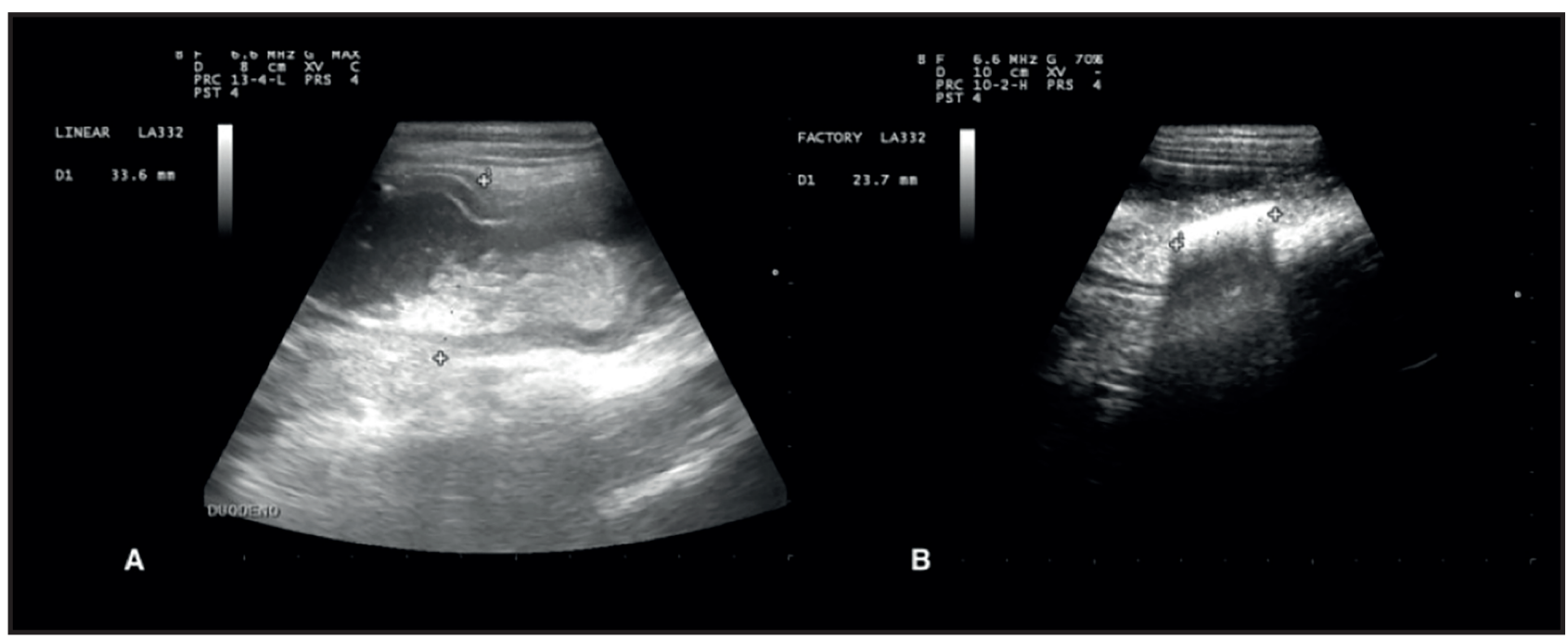

Figure 1. Ultrasonography images of the duodenum. A- Note a marked distended segment of duodenum with fluid and heterogeneous ingesta, associated a thickened wall. B- A hyperechoic structure producing acoustic shadowing between the markers, measuring $2.4 \mathrm{~cm}$ in length, compatible with a small foreign body. 


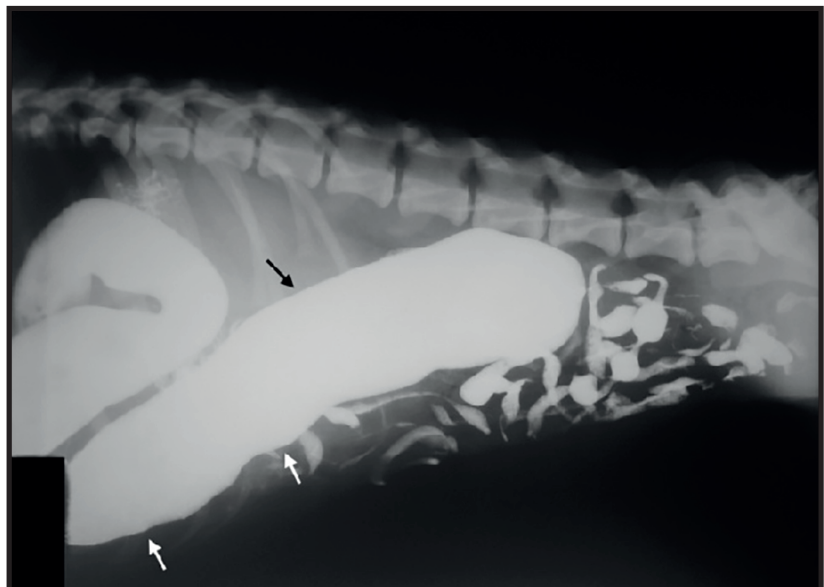

A

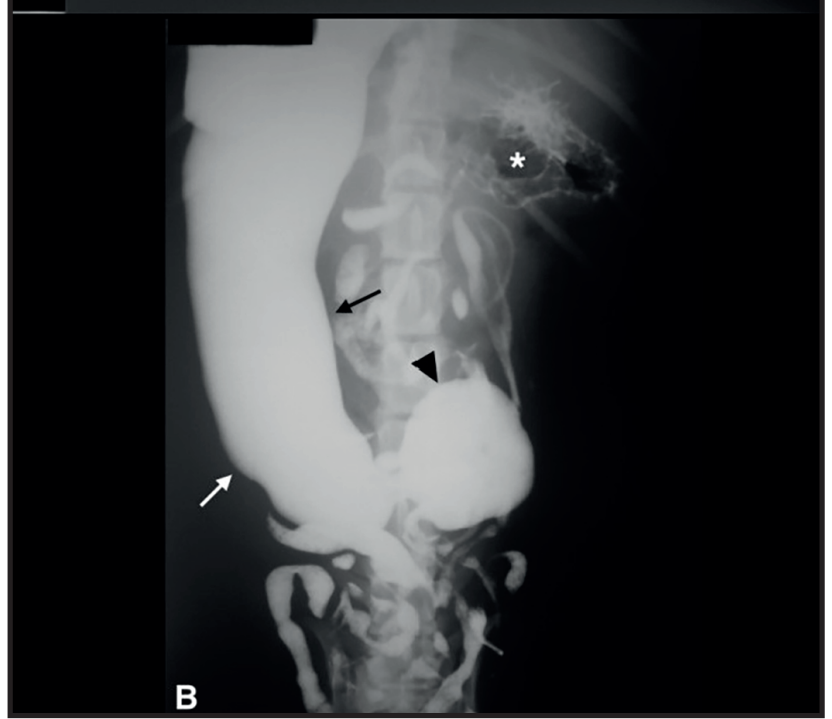

Figure 2. Lateral (A) and ventrodorsal (B) views 60 min after barium administration. Note the persistence of contrast in the dilated and tortuous segment of the duodenum (black and white arrows) and a pocket of bowel at the duodenal caudal flexure diverticulum (arrowhead). Jejunum is filled with a contrast medium, with normal diameter and distribution. Stomach is empty (asterisk).

flexure was connected to a large barium filled saccular structure that measured approximately $7 \mathrm{~cm}$ in diameter, consistent with a duodenal diverticulum. The jejunal loops were normal, with uniform diameter and distribution. The stomach was almost empty with a left displacement of pylorus (Figure 2).

A blood transfusion was performed, and a laparotomy was indicated. The diverticulum, projected from the antimesenteric border of the duodenum, and a small portion of the caudal duodenal segment were resected and an end-side enteroanastomosis was performed, due to the difference in diameter between duodenum and jejunum (Figure 3). Post surgical analgesia was assured by giving tramadol ${ }^{1}[2 \mathrm{mg} / \mathrm{kg} / \mathrm{PO}]$ and dipyrone $^{2}[25 \mathrm{mg} / \mathrm{kg} / \mathrm{PO}$, twice a day. The dog was also medicated with sucralfate ${ }^{3}[12.5 \mathrm{mg} / \mathrm{kg} / \mathrm{PO}]$, ranitidine ${ }^{4}$

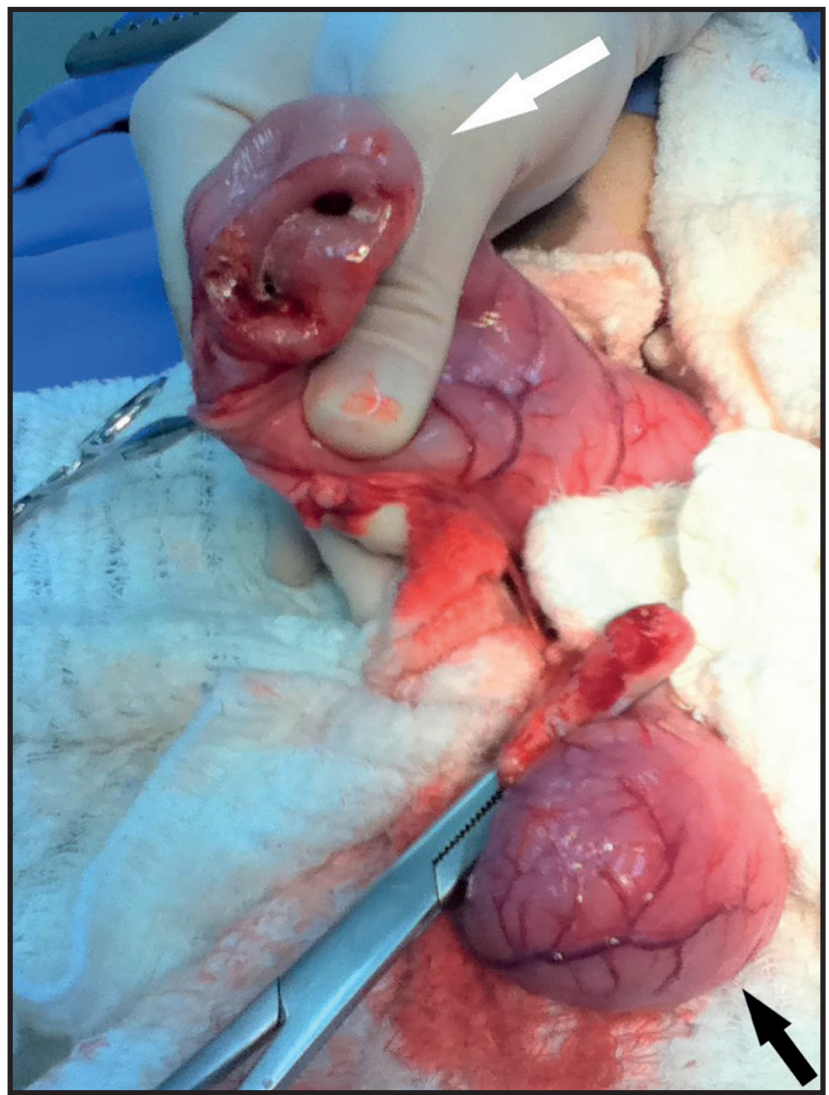

Figure 3. Intraoperative view of the enterectomy site showing duodenal diverticulum (black arrow) and proximal duodenum (white arrow).

[2 mg/kg/PO] and metronidazole ${ }^{2}$ [25 mg/kg/PO] twice daily, and recovered from surgery without complications. In follow-up evaluations no recurrence of clinical signs was observed.

\section{DISCUSSION}

Congenital duodenal diverticulum is a rare condition and it has been already reported in four dogs, interestingly, all Boxers $[3,13,16]$, however, there are reports of jejunal [4] and rectal [9] diverticulum involving other breeds.

The clinical signs of these four cases of duodenal diverticulum $[3,13,16]$ were similar to those found in this dog, including melena, anorexia, vomiting, anemia and syncope. Melena and anemia were likely caused by the bleeding process due to inflammation, ulcerations and rupture of superficial blood vessels in consequence of food stasis in duodenum and diverticulum.

None of the reported cases in literature had the diagnosis of duodenal diverticulum made exclusively by ultrasonography. Other diagnostic imaging modalities, such as gastrointestinal barium study or 
endoscopy, were necessary to confirm the diagnosis $[13,16]$. In one case diagnostic was made during exploratory laparotomy secondary to a foreign body [3].

The marked dilatation of the intestinal segment filled with ingesta impaired ultrasound evaluation, allowing recognition of an obstructive pattern, not the diverticulum itself, in this case. Histopathological examination of the diverticular tissue showed signs of chronic inflammation and a hypertrophied muscle layer of the intestinal wall, characterizing a true duodenal diverticulum. The location (caudal flexure of the duodenum), breed (Boxer) and age (immature) of the dogs affected with duodenal diverticulum were similar in all veterinary cases reported in literature. Dogs presenting signs of gastrointestinal disease such as vomits, regurgitation, anorexia and abdominal pain are common in patients referred to ultrasound examination. However, despite the rare reports described, we must consider this affection as a differential diagnosis, whenever Boxer puppies present these clinical signs associated with gastrointestinal bleeding and syncope.

\section{MANUFACTURERS \\ ${ }^{1}$ Cristália Produtos Químicos Farmacêuticos Ltda. Itapira, SP, Brazil. \\ ${ }^{2}$ Sanofi Aventis Farmacêutica Ltda. Suzano, SP, Brazil. ${ }^{3}$ EMS S/A. Hortolândia, SP, Brazil. \\ ${ }^{4}$ Glaxosmithkline Brasil Ltda. Rio de Janeiro, RJ, Brazil.}

Acknowledgments. The authors would like to thank $\mathrm{PhD}$ Professor Stefano Hagen (University of São Paulo) for his valuable and constructive comments and expertise during article revision and to $\mathrm{PhD}$ Professor Rodolfo Nurmberger Jr., in memoriam, for his knowledge in histopathology.

Declaration of interest. The authors report no conflicts of interest. The authors alone are responsible for the content and writing of the paper.

\section{REFERENCES}

1 Angus L., Larson B., Egodage T. \& Raju S.G. 2013. Traumatic blow out of a duodenal diverticulum: a rare clinical finding. Injury Extra. 44(10-12): 95-98.

2 Bittle M.M., Gunn M.L., Gross J.A. \& Rohrmann C.A. 2012. Imaging of duodenal diverticula and their complications. Current Problems in Diagnostic Radiology. 41(1): 20-29.

3 Blesch M., Livet V., Cabom Q. \& Cachon T. 2016. Obstruction intestinale par un corps étranger au sein d'un diverticule duodénal chez un chiot Boxer. Revue de Médecine Vétérinaire. 167(3-4): 71-76.

4 Buote N.J., Kovak J.R., Fischetti A.J. \& Monette S. 2007. What is your diagnosis? Mineralized-opaque-rimmed soft tissue mass in the right caudoventral portion of the abdomen. Journal of American Veterinary Medical Association. 231(4): 527-528.

5 Chambenois E., Derhy S. \& Arrive L. 2015. Intraluminal duodenal diverticulum. A rare cause of recurrent acute pancreatitis. Clinics and Research in Hepatology and Gastroenterology. 39(3): 278-279.

6 Eusebio M., Ramos A. \& Guerreiro H. 2016. Intraluminal duodenal (windsock) diverticulum: a rare cause of gastrointestinal bleeding. Portuguese Journal of Gastroenterology. 23(2): 113-115.

7 Glener J., Poris S., Foles B. \& Harmon R. 2016. Perforated duodenal diverticulum case report. International Journal of Surgery Case Reports. 29: 100-102.

8 Kassir R., Boueil-Bourlier A., Baccot S., Abboud K., Dubois J., Petcu C.A., Boutet C., Chevalier U., Montveneur M., Cano M.I., Ferreira R., Debs T. \& Tiffet O. 2015. Jejuno-ileal diverticulitis: etiopathogenicity, diagnosis and management. International Journal of Surgery Case Reports. 10: 151-153.

9 Mehrjerdi H.K., Mirshahi A. \& Afkhami A. 2013. Rectal diverticulum in a Terrier dog: a case report. Veterinary Research Forum. 4(1): 63-67.

10 Penades M., Guerrero I., Peña A.B. \& Corpa J.M. 2010. Duodenal gland cysts and pseudodiverticula in sheep. Journal of Veterinary Diagnostic Investigation. 22(4): 649-651.

11 Peng H.L., Su C.T., Chang C.Y. \& Lau B.H. 2014. Intraluminal duodenal diverticulum in a child concomitant with an entrapped coin and a duodenal polyp. Formosan Journal of Surgery. 47(6): 236-239.

12 Perrot T., Poletti P.A., Becker C.D. \& Platon A. 2012. The complicated duodenal diverticulum: retrospective analysis of 11 cases. Clinical Imaging. 36(4): 287-294.

13 Polf H. \& Poteet B. 2010. Imaging diagnosis - duodenal diverticulum in a dog. Veterinary Radiology \& Ultrasound. 51(1): 61-64.

14 Schroeder T.C., Hartman M., Heller M., Klepchick P. \& Ilkhanipour K. 2014. Duodenal diverticula: potential complications and common imaging pitfalls. Clinical Radiology. 69(10): 1072-1076. 
C.A.B. Lorigados, A.M. Zoppa, A. Schiller, et al. 2021. Partial Obstruction and Intestinal Bleeding Secondary to a Congenital Duodenal Diverticulum in a Dog. Acta Scientiae Veterinariae. 49(Suppl 1): 597.

15 Simões V.C., Santos B., Magalhães S., Faria G., Silva D.S. \& Davide J. 2014. Perforated duodenal diverticulum: surgical treatment and literature review. International Journal of Surgery Case Report. 5(8): 547-550.

16 Van Klaveren N.J., Grinwis G.C.M., Brocks B.A.W. \& Kirpensteijn J. 2008. Collapse following gastrointestinal bleeding secondary to a congenital duodenal diverticulum in two littermate boxer pups. Journal of Small Animal Practice. 49(2): 103-106. 